\title{
Diseño de Juegos Basados en el Paradigma de Gramáticas Artificiales Para Favorecer el Aprendizaje Implícito en Niños
}

\section{Design of Games Based on the Paradigm of Artificial Grammars to Facilitate Implicit Learning in Children}

\author{
Carolina Thibaut y Ricardo Rosas \\ Pontificia Universidad Católica de Chile
}

\begin{abstract}
Se desarrollaron dos juegos en formato computacional basados en el paradigma de gramáticas artificiales de Reber, con el objetivo de favorecer la demostración de aprendizaje implícito en niños con distintas características: normales $(\mathrm{N})$, con déficit atencional (DA) y con déficit intelectual (DI). Los resultados muestran que los niños N y DA rinden por sobre lo esperado por azar, a diferencia de los DI quienes logran un desempeño similar al azar. Los hallazgos sugieren que niños con problemas de atención aprenden a través de vías implícitas al igual que niños sin problemas de atención, independientemente del juego utilizado.
\end{abstract}

Palabras Clave: gramática artificial, aprendizaje implicito, juego.

Two computer-based games were developed based on Reber's Artificial Grammar Paradigm, in order to facilitate implicit learning ocurrence in normal $(\mathrm{N}), \mathrm{ADHD}$, and intellectually disabled (ID) children. Results show that $\mathrm{N}$ and ADHD children perform above chance level, as opposed to ID children whose performance is equivalent to random response. These findings show that ADHD children learn through implicit paths as well as children without attention problems, independently of the game used.

Keywords: artificial grammar, implicit learning, game.

A través del aprendizaje implícito las personas logran aprender reglas abstractas sin que estos conocimientos sean accesibles a su conciencia. Este aprendizaje ocurre de manera incidental (Seger, 1994), esto es, ocurre independientemente de los intentos deliberados o concientes que se realicen por aprender.

Lamentablemente, los estudios en niños son escasos y los resultados no son consistentes respecto a la ocurrencia de aprendizaje implícito en esta población, por lo que en esta investigación se intenta resolver bajo qué condiciones es posible demostrar la ocurrencia de aprendizaje implícito en niños. A continuación se profundiza en los antecedentes asociados a esta situación.

Una de las dificultades radica en la complejidad del paradigma experimental clásico en el que se

Carolina Thibaut Páez, Programa de Magíster en Psicología Educacional, Pontificia Universidad Católica de Chile.

Ricardo Rosas Díaz, Escuela de Psicología, Pontificia Universidad Católica de Chile.

La correspondencia relativa a este artículo deberá ser dirigida a los autores a Escuela de Psicología, Pontificia Universidad Católica de Chile, Vicuña Mackenna 4860, Santiago, Chile. E-mail: cthibaut@uc.cl; rrosas@uc.cl

Este estudio fue financiado por el Proyecto Fondecyt $\mathrm{N}^{\circ}$ 1050886 “ ¿Son la memoria de trabajo y la atención necesarias para el aprendizaje implícito? Evidencia con niños con déficit intelectual y déficit atencional". sustenta: el paradigma de gramáticas artificiales de Reber (1967), cuya estructura es posible observar en la Figura 1.

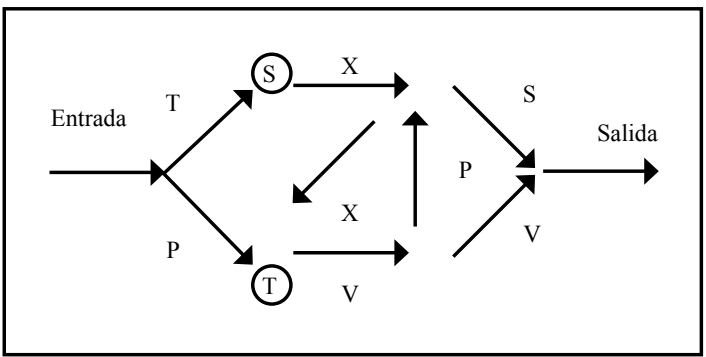

Figura 1. Estructura clásica de gramática artificial. Se forman palabras siguiendo siempre las siguientes reglas: (a) se comienza siempre por la entrada, (b) se termina siempre en la salida, (c) se sigue el orden de las flechas, (d) las letras en círculo se pueden omitir o repetir.

El experimento clásico de gramáticas artificiales incluye dos momentos: se comienza con una fase de entrenamiento o aprendizaje, y luego, se pasa a una segunda fase de prueba o evaluación. En la fase de entrenamiento las personas son expuestas a series de ordenaciones particulares de una gramática artificial que deben memorizar (Reber, 1993). Los experimentos de Reber (1967), le llevaron a descubrir que los sujetos que son expuestos sólo a ordenaciones 
"legales", muestran una ventaja significativa en su capacidad para memorizar los ítemes, respecto a un grupo control expuesto a ordenaciones aleatorias. Este importante hallazgo, fue el que le llevó a sugerir la presencia de aprendizaje (implícito) a partir de las regularidades gramaticales en adultos.

La fase de evaluación, por su parte, consiste en informar a los sujetos que los ejemplares vistos en el entrenamiento corresponden a una estructura reglada, por lo que se les solicita que distingan de un pool de ejemplares distintos, cuáles de ellos pertenecen a estructuras gramaticales y cuáles pertenecen a estructuras no gramaticales, según respeten o no dichas reglas. De esta forma se busca medir el aprendizaje de los patrones subyacentes a las ordenaciones expuestas durante la fase de entrenamiento (Sun, 1998). La realización de esta última tarea corresponde a la prueba crítica que permite evaluar el logro de aprendizajes. A través de ella, se ha demostrado que los sujetos reconocen acertadamente la gramaticalidad de los nuevos ítemes, con niveles de acierto de entre $60 \%$ y $80 \%$, aunque son incapaces de verbalizar las reglas abstractas, o bien, reportan reglas erróneas (Augart, 1994).

\section{Hallazgos Divergentes Sobre Aprendizaje Implícito en Niños}

Es muy probable que la escasa investigación en este tema se deba a la gran dificultad que tiene implementar el paradigma de gramáticas artificiales en niños, ya que implica tareas difíciles de realizar para ellos. No obstante, y a pesar de las dificultades recién mencionadas, se han realizado algunas interesantes investigaciones que han intentado adaptar el paradigma experimental de gramáticas artificiales para demostrar aprendizaje implícito en este grupo.

Uno de los trabajos pioneros en esta área consiste en un estudio realizado por Roter (1985 citado en Perruchet \& Vinter, 1998), el cual no encontró diferencias significativas en la ejecución de la prueba de gramaticalidad en función de la edad. Para ello, el autor comparó grupos de niños de 6-7 años, 9-10 años y 12-15 años.

Gómez y Gerken (1999) realizaron una adaptación del paradigma clásico de gramáticas artificiales para niños cercanos al año de edad. Se expuso a los niños a algunas ordenaciones auditivas generadas a partir una gramática artificial finita de sílabas sin sentido. Para evaluar los aprendizajes, se le decían dos ordenaciones a los niños: una gramaticalmente legal y otra ilegal. Los niños se orientaron por más tiempo hacia las ordenaciones correctas, lo que indica que los niños desde muy pequeños son sensibles al aprendizaje implícito.

Por su parte, Rosas, Condemarín, Nussbaum y Sun (1999) adaptaron el paradigma de gramáticas artificiales a un juego para niños, administrado mediante una plataforma computacional de videojuegos portátiles. Aunque no se obtuvieron resultados concluyentes sobre aprendizaje implícito en niños en edad escolar, se realizó un aporte importante respecto al formato de presentación. Aparte del uso de una herramienta tecnológica, por primera vez se incorporó el juego como una alternativa para adaptar el complejo paradigma reberiano a las características de los niños.

A partir de esta experiencia, Rosas, Condemarín, Escobar, Sun y De Padua (1999), aplicaron estos juegos a adultos, quienes sí rindieron significativamente por sobre lo esperado por azar. Estas evidencias llevaron a plantear diferencias en el aprendizaje implícito, según la edad o nivel de desarrollo.

Rosas y Grau (2002), evaluaron el rol de la memoria de trabajo en el aprendizaje implícito, mediante la comparación de la ejecución de tres tareas diferentes de aprendizaje implícito (dos basadas en el paradigma de gramáticas artificiales de Reber y uno basado en el paradigma de covariaciones de Lewicki, Czyzewska y Hoffman (1986) ${ }^{1}$ en dos grupos de niños: niños con CI normal y una buena ejecución en pruebas de memoria de trabajo y niños con déficit intelectual y un pobre desempeño en pruebas de memoria de trabajo. Con el objetivo de hacer más comprensible la tarea de gramáticas artificiales a niños pequeños, los autores desarrollaron una situación estructuralmente idéntica a la reberiana, pero diferente en contenido. Estos autores lograron demostrar la mayor robustez del paradigma de gramáticas artificiales, pero no consiguieron demostrar de manera fehaciente la ocurrencia de aprendizaje implícito en niños. Uno de los problemas de este estudio fue la evaluación de los niños con retardo intelectual, ya que a pesar de que comprendieron y ejecutaron bien la tarea de aprendizaje implícito presentada en un formato de juego, tuvieron grandes dificultades para comprender la naturaleza de la tarea de evaluación.

Finalmente, en un reciente estudio, López (2005) desarrolló una adaptación del paradigma de gra-

\footnotetext{
Los estudios de aprendizaje de covariaciones demuestran aprendizaje implícito como una covariación entre formas dentro de un estímulo visual o entre formas de un estímulo visual y un nivel verbal (Seger, 1994).
} 
máticas artificiales a dos formatos de presentación para los estímulos: uno alfabético y otro figurativo. Estos formatos se aplicaron a niños de 8 años, demostrándose ocurrencia significativa de aprendizaje implícito por sobre el azar y ausencia de diferencias significativas entre ambos formatos de presentación. Aunque esta investigación muestra resultados contundentes a nivel empírico, la actividad resulta difícil de ser replicada por niños en situaciones no experimentales, ya que carece de elementos lúdicos que incentiven la realización de la tarea de manera autónoma y espontánea.

Estos hallazgos muestran las discrepancias que existen respecto a los resultados y dejan en evidencia lo difícil que es lograr demostrar mediante pruebas inequívocas y confiables la ocurrencia de aprendizaje implícito en esta población. Además, se dejan en evidencia las serias dificultades metodológicas, ya que no existen aún instrumentos suficientemente adecuados para evaluar la adquisición de estructuras complejas a través de paradigmas experimentales en niños.

\section{El Uso del Juego Como Alternativa Para el Aprendizaje Implícito en Niños}

El juego corresponde a la actividad que los niños realizan por excelencia por lo que se presta como una buena alternativa para presentar los estímulos de un paradigma de gramáticas artificiales de manera cercana y familiar. El juego puede servir de soporte "intermediario" entre la tarea experimental y los intereses del niño, ya que por medio de un contexto lúdico, es posible encubrir la fase de memorización que realizan los adultos en la situación experimental de gramáticas artificiales.

Por otra parte, aunque los requisitos atencionales del aprendizaje implícito no están totalmente claros, en general se acepta que la atención focalizada de los sujetos hacia los estímulos de interés es una condición necesaria en el paradigma de gramáticas artificiales (Berry \& Cock, 1998 citados en Sun, 2000). ¿Y cómo focalizar análogamente la atención de los niños en esta tarea? Una alternativa es el diseño de un juego que cumpla con los requisitos de atractivo conocidos para este tipo de actividades.

De acuerdo a la literatura, hay elementos en los juegos que explican su atractivo para los niños: (a) la existencia de un personaje (protagonista) con el cual los niños puedan identificarse, que no les provoque rechazo y que facilite el logro de los objetivos del juego (Malone \& Lepper, 1987); (b) la presencia de múltiples personajes, especialmente antagonistas, los cuales van apareciendo en la medida que se va avanzando a través de los niveles del juego, así como la exposición del jugador a crecientes desafíos (Kafai, 1997 citado en Rosas et al. 2000); (c) la existencia de un desafío juega un rol decisivo en los videojuegos, así como el entregar feedback permanente, aunque los avances sean insignificantes (Klawe, 1998; Malone \& Lepper, 1987); (d) la presencia de un aspecto de la fantasía y curiosidad hace de los juegos herramientas intrínsecamente motivadoras (Baltra, 1990; Malone \& Lepper, 1987), (e) la creación de un mundo interesante, que permita al jugador insertarse en la dinámica del juego, y cumplir con las tareas y metas de éste (Baques, 1995 citado en Stewart \& Kowaltzke, 1997). Esto permitiría crear un efecto de inmersión, estado en el cual el niño se involucra y concentra en las reglas y dinámica del juego para poder cumplir exitosamente el objetivo de éste, donde el propósito central no es aprender, sino jugar (Hubbard, 1991 citado en Rosas et al., 2000).

Todo lo anterior contribuye a pensar que es posible traducir el paradigma de gramáticas artificiales a un formato lúdico que permita evaluar la ocurrencia de aprendizaje implícito en niños. Se reconoce que ésta no es una idea nueva, ya que otros autores (López, 2005; Rosas, Condemarín, Nussbaum \& Sun, 1999; Rosas \& Grau, 2002) han realizado adaptaciones de la gramática artificial a formatos lúdicos, sin embargo, los resultados no han sido los esperados: las dos primeras investigaciones no consiguieron demostrar de manera fehaciente la ocurrencia de aprendizaje implícito en los niños y la última perdió de vista los aspectos lúdicos de la actividad. Por tanto, el objetivo principal de este trabajo consiste en la creación de un juego, basado en el paradigma de gramáticas artificiales de Reber, para niños de entre 6 y 8 años de edad y que favorezca la demostración de aprendizaje implícito en niños.

\section{Método}

\section{Etapas de la Investigación}

La investigación contempló tres etapas que se llevaron a cabo de manera consecutiva:

Etapa 1: Aproximación a los juegos actuales de los niños entre 6 y 8 años. Se seleccionaron intencionadamente tres cursos mixtos de niños de $1^{\circ}$ básico, que pertenecían a establecimientos de distinta dependencia: municipal, particular-subvencionada y particular pagada de la Región Metropolitana. Participaron todos los alumnos presentes en cada curso. 
Tabla 1

\section{Composición de la muestra}

\begin{tabular}{lllll}
\hline Grupo & Condiciones & Hombre & Mujer & Total \\
\hline 1 & Niños Normales (N) & 2 & 6 & 8 \\
2 & Niños con déficit atencional (DA) & 4 & 4 & 8 \\
3 & Niños con déficit intelectual (DI) & 4 & 4 & 8 \\
Total & & 10 & 14 & 24 \\
\hline
\end{tabular}

También participó un grupo mixto de niños con retardo mental, con edad mental entre 6 y 8 años, que pertenecían a una escuela especial particular-subvencionada para niños con deficiencia mental.

Se realizó una entrevista a cada grupo de niños, respetando la conformación natural de los cursos en los respectivos establecimientos. Cada una de estas correspondió a una entrevista lúdica en la cual se plantearon distintas situaciones hipotéticas y los niños debían responder señalando a viva voz los juguetes, juegos de recreo, personajes de dibujos animados y juegos a los que más les gusta jugar. Finalmente debían realizar un dibujo con el elemento lúdico preferido.

En esta etapa se realizaron análisis cualitativos basados en categorías emergentes y en tablas de frecuencia para poder obtener información tanto de la diversidad de respuestas, como de los elementos lúdicos más recurrentes entre los niños.

Etapa 2: Diseño y desarrollo de un formato lúdico basado en el paradigma de gramáticas artificiales. En base a los juegos y elementos lúdicos que los niños señalaron como sus preferidos en la primera etapa, se seleccionaron aquellos aspectos de los juguetes, juegos y dibujos animados que los niños señalaron con mayor frecuencia, considerando también las variables de género y tipo de dependencia. A continuación se trabajó en el diseño de un guión gráfico y de una metáfora lúdica que integrara los elementos de la gramática artificial y los elementos lúdicos recolectados en las entrevistas, para facilitar la presentación de los estímulos ${ }^{2}$

Etapa 3: Prueba piloto. La muestra estuvo constituida por tres grupos de niños que cursaban $1^{\circ}$ y $2^{\circ}$ básico en un establecimiento municipal de la comuna de Quilicura. Las edades de los niños fluctuaban entre los 6 y los 9 años, con un promedio de 7 años de edad cronológica. Los grupos se constituyeron de la siguiente manera (Tabla 1):

Para la selección de los niños se aplicaron el Test WISC III, normas chilenas 2004 (Ramírez \& Rosas, 2007) para la evaluación de nivel intelectual; y el Test de Conners abreviado para profesores, para la evaluación de déficit atencional.

Los niños calificados como normales son aquellos que presentaron un CI entre normal lento y normal alto en el WISC III y que obtuvieron puntajes iguales o menores a 14 puntos en el Test de Conners.

Los niños calificados con déficit atencional obtuvieron puntajes pertenecientes al mismo rango de los niños normales en el WISC III; y presentaron puntajes superiores a 14 puntos, correspondientes a síndrome de déficit atencional moderado a severo en el Test de Conners.

Los niños con déficit intelectual, en tanto, se ubicaron dentro de las categorías limítrofe y retardo mental en el WISC III y obtuvieron hasta 14 puntos en el Test de Conners.

2 La descripción del formato lúdico creado se presenta en el apartado Los Juegos.
Una vez evaluados, se invitó a los niños seleccionados a jugar de manera individual con un computador portátil. Cada niño tuvo una sesión de juego que constó de la aplicación de dos juegos en orden consecutivo y de manera contrabalanceada: Trenes y Pesca. El juego Trenes, corresponde a una adaptación del juego creado por Grau (2002) para ser utilizado en un formato electrónico. Este fue diseñado para evaluar aprendizaje implícito en niños con retardo mental y cumple con los requisitos del paradigma de gramáticas artificiales, sin embargo, su presentación no resulta lúdica para los niños, ya que es una prueba bastante monótona. Por este motivo, se utilizó como una herramienta de comparación para poder evaluar tanto aprendizaje implícito, como la influencia de los aspectos lúdicos. El segundo corresponde al juego creado a partir de las entrevistas grupales realizadas a los niños en la Etapa 1.

Al finalizar ambos juegos se aplicó un breve cuestionario con el propósito de que los niños evaluaran comparativamente los aspectos lúdicos ya mencionados.

\section{Los Juegos}

En los dos juegos se presentan distintos animales que reemplazan a cada una de las letras utilizadas en el paradigma de gramáticas artificiales (ver Figura 2). Cada uno de estos animales se eligió por su diversidad de colores y formas, para que fueran claramente diferenciables por los niños.

El procedimiento siguió la estructura básica del paradigma de gramáticas artificiales, presentando una fase de entrenamiento o de aprendizaje, y a continuación, una fase de evaluación de aprendizaje implícito.

Fase de aprendizaje. En esta tarea, el niño debe recordar el orden en que se presentan los animales. En el caso de Trenes los animales se encuentran en vagones de un tren; en el caso de Pesca corresponde al orden en que los clientes solicitan su pedido en un restaurante. Una vez que el niño ha aprendido el orden en que se presenta el ejemplar, debe reconstruirlo respetando el orden presentado (Ver Figuras 3 y 4). Si lo hace correctamente, continúa con la siguiente ordenación. Si el ejemplar reconstruido es incorrecto, en la pantalla aparece la ordenación correcta para que el niño la copie.

En esta fase los niños realizan 16 ordenaciones de distinto largo, las cuales fueron creadas en base a la gramática artificial y corresponden al número máximo de ejemplares que se pueden crear con estos elementos para conformar ordenaciones que no tengan un largo menor a 3, ni mayor a 6 elementos. De esta manera se propició el mayor número de ejemplares posibles para que el niño logre aprender la estructura, con una cantidad de elementos adecuados para ser memorizados.

Fase de evaluación. Luego de finalizada la fase de aprendizaje, se realizó la evaluación de aprendizaje implícito, en la que se presentaron simultáneamente dos ordenaciones de animales en la pantalla del computador (trenes o bandejas con platos) y 


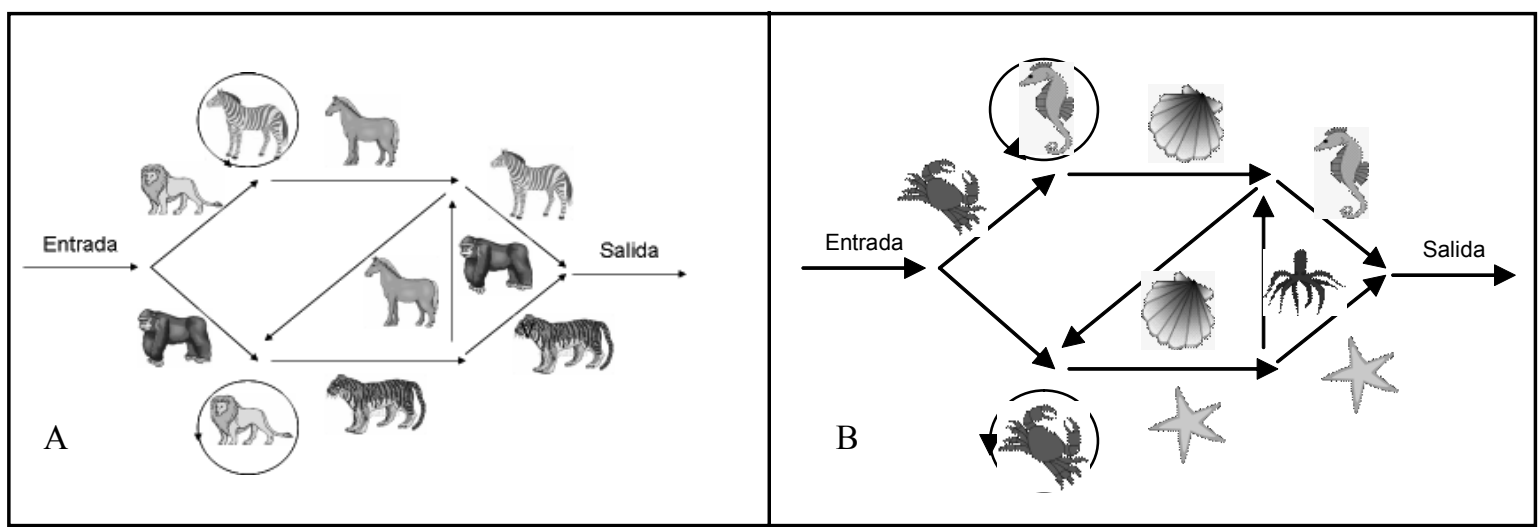

Figura 2. A. Gramática artificial con los animales usados en Trenes. B. Gramática artificial con los animales usados en Pesca.

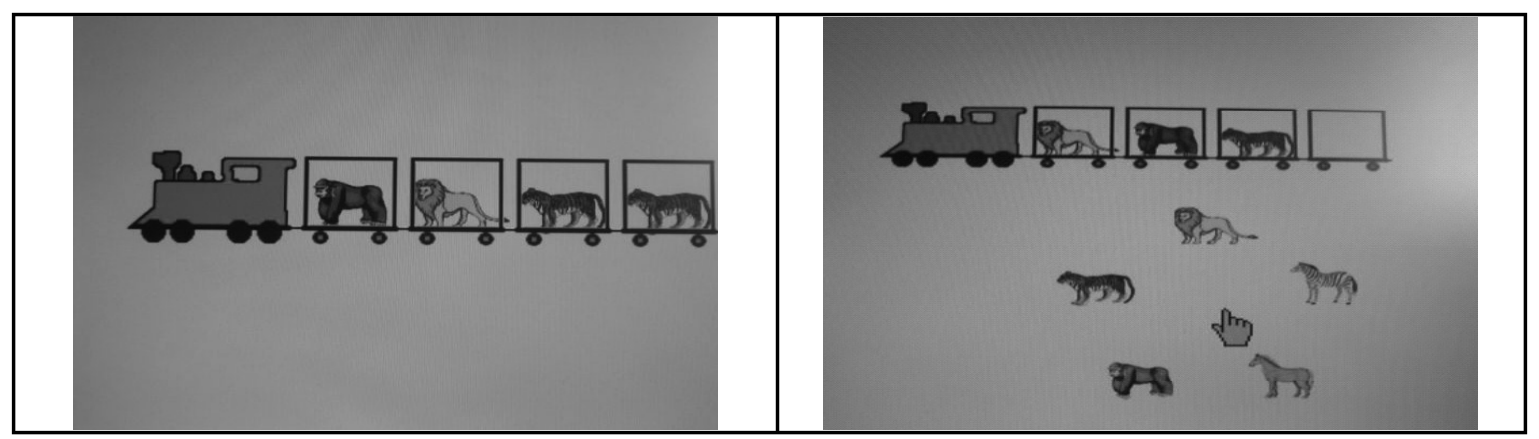

Figura 3. Ejemplar usado en Trenes: los niños deben memorizarlo y reconstruirlo en la siguiente pantalla, eligiendo los animales en el mismo orden en que fueron presentados.

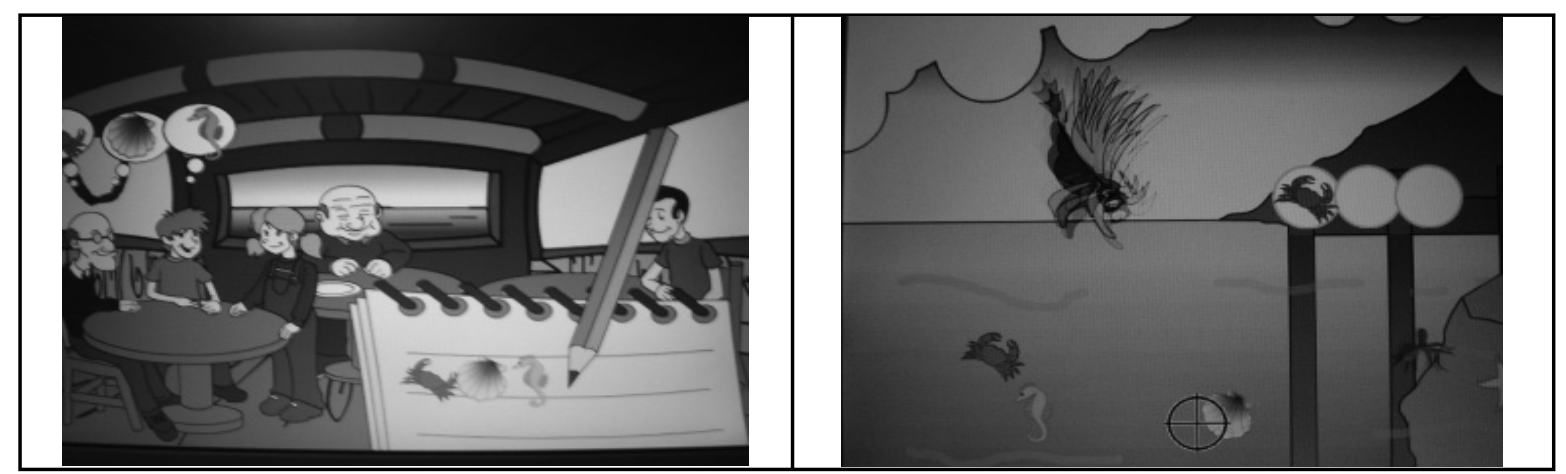

Figura 4. Ejemplar usado en Pesca: los niños deben memorizarlo y reconstruirlo en la siguiente pantalla, eligiendo los animales en el mismo orden en que fueron presentados.

el niño debía seleccionar la ordenación correcta marcando sobre ella con el mouse.

Se incluyeron 11 ítemes, conformados por una ordenación correcta (target) y un distractor. Estos últimos se crearon en base a ciertos criterios: que los animales correspondieran a los utilizados en las ordenaciones válidas, que el animal con que comienza y termina la ordenación correspondiera a los indicados por la gramática y que todas las ordenaciones tuvieran el mismo número de elementos.

Dado que en la fase de aprendizaje se utilizaron todas las ordenaciones posibles entre 3 y 6 elementos, para la fase de evaluación se utilizaron sólo ordenaciones de una extensión fija de 7 elementos cada uno, tanto para los ejemplares válidos como para los distractores. 


\section{Resultados}

\section{Aprendizaje Implícito}

Se compararon los puntajes promedio que obtuvieron los niños en la evaluación de aprendizaje implícito en Trenes y en Pesca con el puntaje esperado por azar (5.5) a través de una prueba $t$ para una muestra. Tal como se observa en la Tabla 2, los niños logran puntajes que superan al azar en el juego Trenes. En cambio, en el juego Pesca, los niños logran una ejecución que no es significativamente distinta al azar.

Se evaluó la magnitud de las diferencias de puntaje promedio de los juegos mediante una prueba $t$ para muestras relacionadas. En esta prueba se pudo observar que pese a que los niños logran en promedio mayor aprendizaje en el juego Trenes que en el juego Pesca, estas diferencias no son significativas $(t=0.510 ; p=0.615)$, lo que evidencia que ambos juegos resultan estímulos semejantes para promover aprendizaje implícito.

Se realizó un análisis por condición en el cual se sumaron los puntajes obtenidos por los niños en ambos juegos, independientemente del orden en el que fueron aplicados. De esta manera se obtuvo un puntaje total de aprendizaje implícito, con un puntaje esperado por azar igual a 11 puntos. En el gráfico (Figura 5) es posible observar que los niños normales (N) y los niños con déficit atencional (DA) rinden por sobre lo esperado por azar. Los niños con déficit intelectual (DI), en cambio, logran un desempeño que es igual a lo esperado por azar.

Se realizó una prueba de análisis de la varianza (ANOVA) para comparar los resultados obtenidos por los tres grupos de niños, y no se encontraron diferencias significativas por condición $(F=2.05$; $p=0.154)$, ver Tabla 3 .

\section{Tabla 2}

Prueba t para una muestra para evaluar aprendizaje implícito en ambos juegos

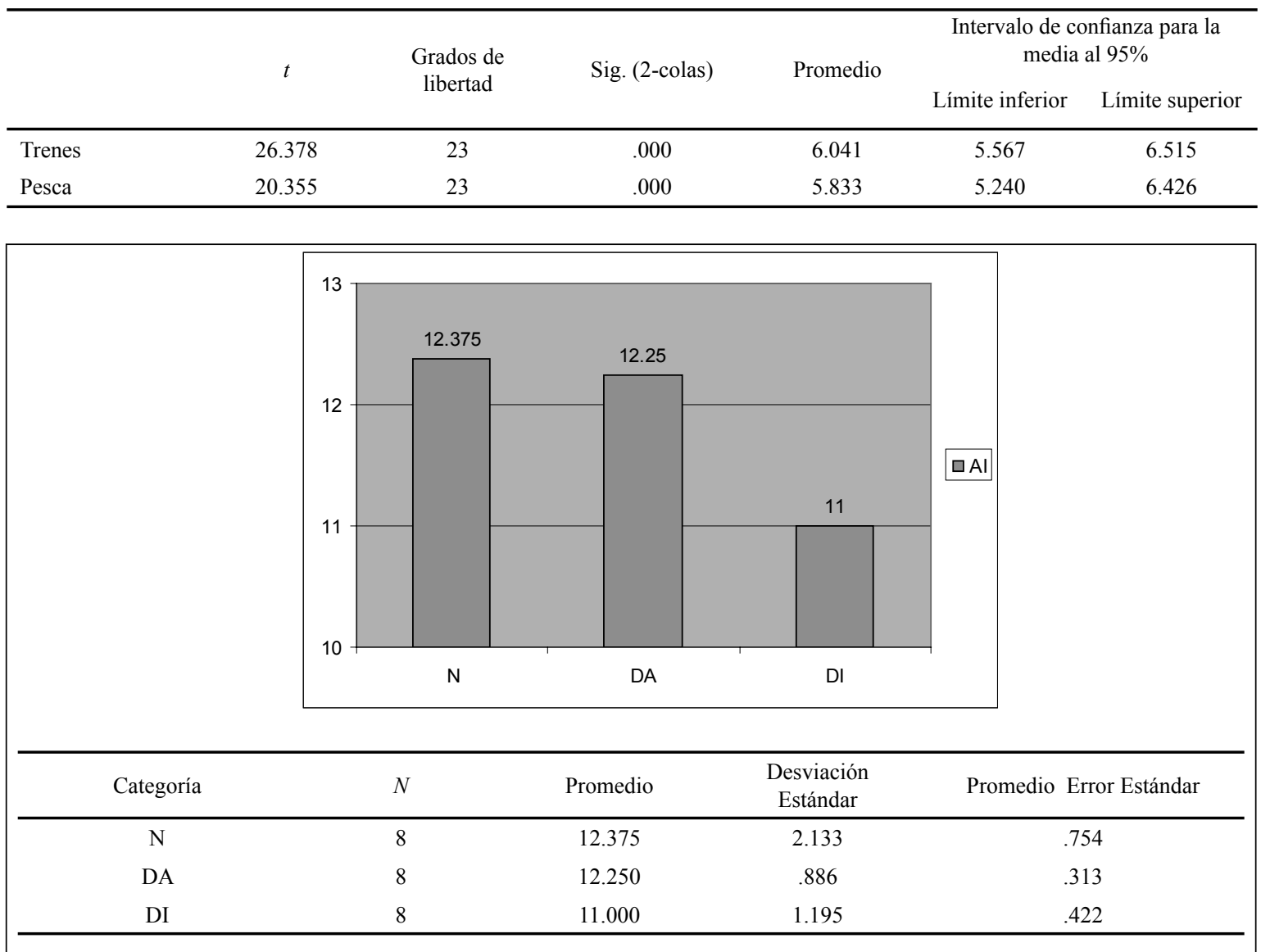

Figura 5. Resultados de aprendizaje implícito por condición. 
Tabla 3

ANOVA para evaluar diferencias de aprendizaje implícito por condición

\begin{tabular}{lccccc} 
& $\begin{array}{c}\text { Suma de cua- } \\
\text { drados }\end{array}$ & Grados de libertad & Promedio & $F$ & Sig. \\
\hline Entregrupos & 9.250 & 2 & 4.625 & 2.050 & .154 \\
Intragrupos & 47.375 & 21 & 2.256 & & \\
Total & 56.625 & 23 & & & \\
\hline
\end{tabular}

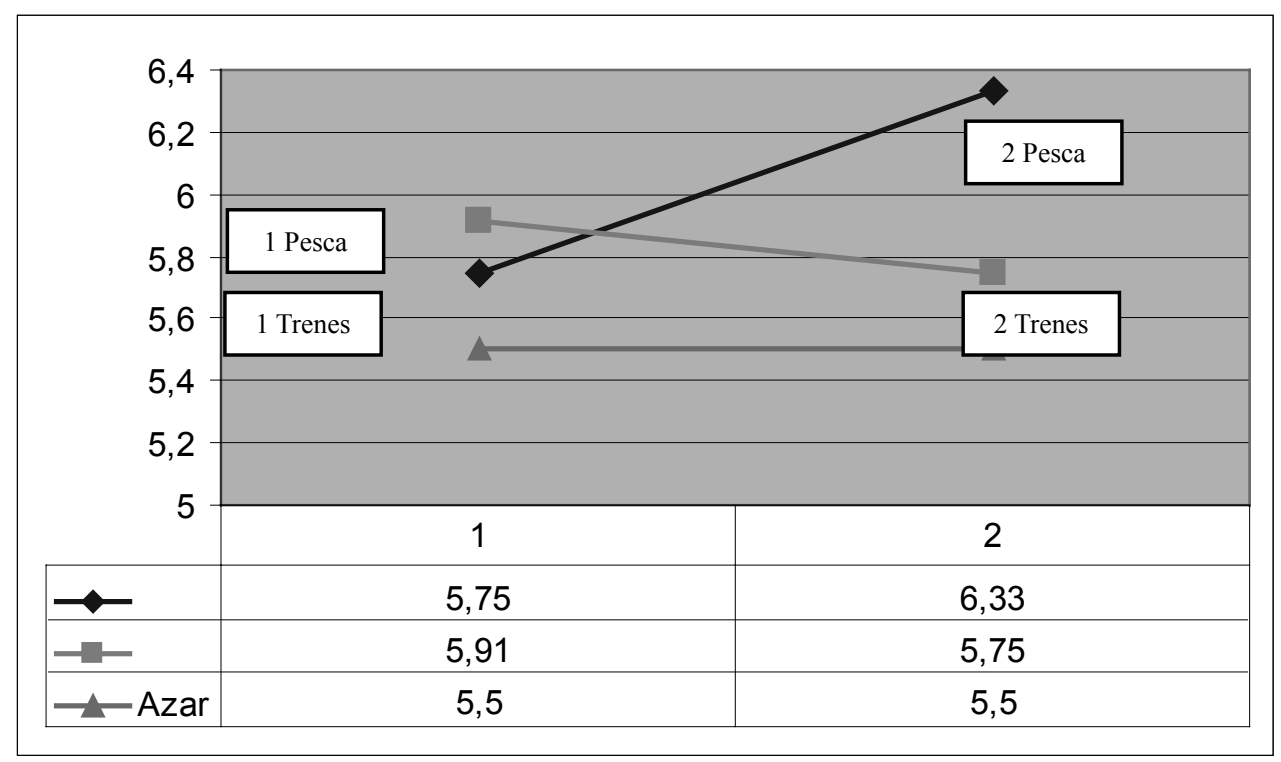

Figura 6. Diferencia de puntaje según el orden de presentación de los juegos: 1 corresponde al juego administrado en primer lugar y 2, al juego administrado en segundo lugar.

\section{Orden de Presentación de los Juegos y Aprendizaje Implícito}

Dado que todos los niños fueron evaluados con ambos juegos de manera consecutiva, se realizaron comparaciones entre los resultados de aprendizaje implícito en función del orden de presentación de los juegos. En la Figura 6 se puede observar que 1 corresponde al juego administrado en primer lugar y 2, al juego administrado en segundo lugar. De esta manera, si el primer juego es Pesca, el segundo corresponde a Trenes y viceversa.

En el gráfico se aprecia que los niños obtienen un mejor desempeño en Pesca independientemente del orden de presentación de los juegos, aunque estas diferencias no son estadísticamente significativas tanto para la aplicación $1(t=-0.873 ; p=0.392)$, como para la aplicación $2(t=-0.528 ; p=0.603)$.

Sin embargo, al contrastar la ejecución de los niños en estos juegos respecto a lo esperado por azar, es posible reconocer que:
1. En la aplicación del juego 1, pese a que los resultados con el juego Pesca son superiores, estos no alcanzan a ser significativamente distintos a los esperados por azar $(t=1.820 ; p=0.082)$. Lo mismo ocurre con Trenes $(t=1.149 ; p=$ 0.263 ).

2. En la aplicación 2, en cambio, los niños obtienen con el juego Pesca un desempeño significativamente superior al esperado por azar $(t=2.106$; $p=0.047)$. En Trenes no existe tal diferencia $(t$ $=0.464 ; p=0.647$ ).

En la Figura 7, por su parte, es posible observar los resultados obtenidos por los niños de los tres grupos en función del orden de presentación de los juegos (juego 1 y 2). Las diferencias entre los resultados obtenidos por los niños de las tres condiciones en el juego 1 no son estadísticamente significativas $(F=0.929 ; p=0.411)$. En el juego aplicado en segundo lugar, en cambio, sí se aprecian diferencias 


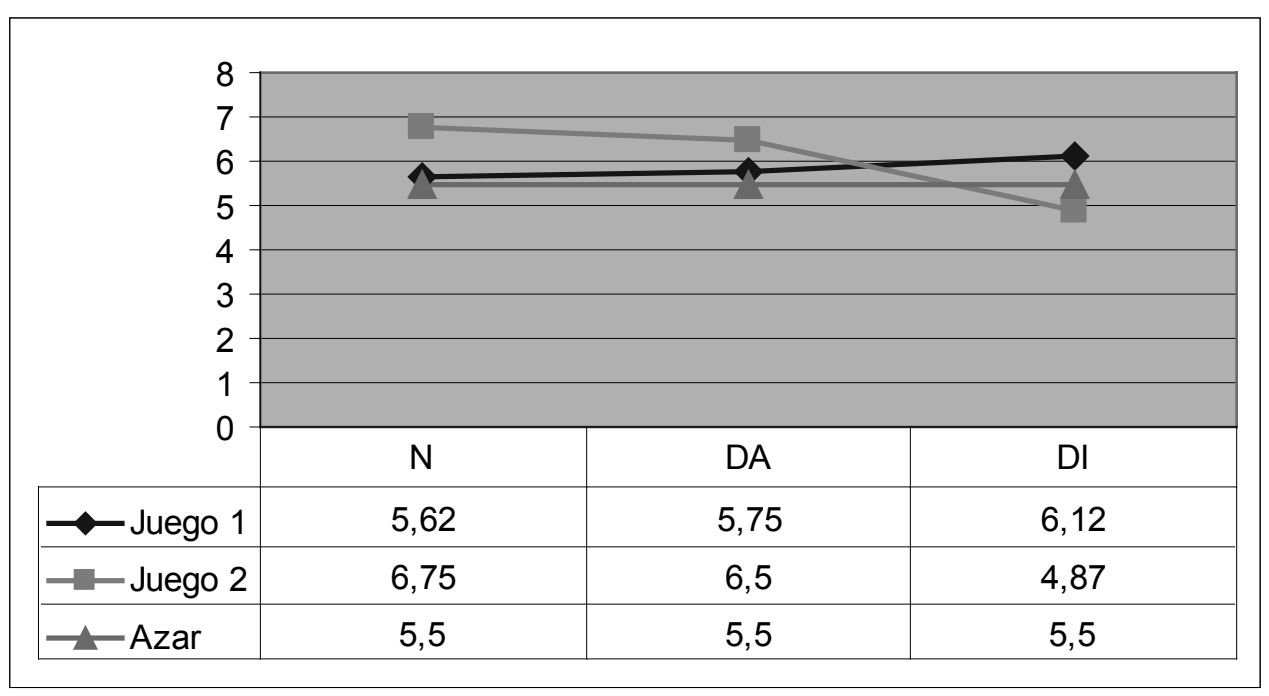

Figura 7. Resultados por condición, en función del orden de presentación de los juegos.

estadísticamente significativas entre los distintos grupos de niños $(F=3.924 ; p=0.036)$.

Las diferencias en el juego 2 se encuentran entre los niños N y DI $(F=1.875 ; p=0.044)$. No se aprecian diferencias estadísticamente significativas entre los niños $\mathrm{N}$ y DA $(F=0.250 ; p=0.937)$ ni entre los niños DA y DI $(F=1.625 ; p=0.088)$.

\section{Aspectos Lúdicos de los Juegos y Aprendizaje Implícito}

Para evaluar los aspectos lúdicos de los juegos se aplicó un breve cuestionario a los niños, donde ellos debían responder a las siguientes preguntas: ¿qué juego te pareció más fácil?, ¿qué juego te pareció más entretenido? y ¿en qué juego te concentraste más? La primera pregunta no se relaciona directamente con las características lúdicas de un juego, sin embargo se incluyó, ya que permite obtener la percepción de los niños respecto a este indicador que actúa como control en la comparación de los juegos.

Los resultados del cuestionario (Figura 8) arrojaron que un $58.33 \%$ de los niños le pareció que el juego Pesca es más fácil. Mientras que un $41.66 \%$ considera lo contrario. Esta diferencia no es estadísticamente significativa $(t=-0.811 ; p=0.426)$.

Un 75\% de los niños considera que Pesca es más entretenido que Trenes (25\%). Esta diferencia sí es significativa $(p=0.023)$.

Por otra parte, un $70.83 \%$ de los niños señaló que se concentró más en Pesca que en Trenes. Esto da cuenta de una diferencia que no alcanza a ser significativa respecto a la percepción que tienen los niños en esta variable $(p=0.064)$.

Considerando que el orden de presentación de los juegos podría tener cierto efecto sobre la percepción de los niños, se realizaron comparaciones para discriminar si el primer juego aplicado tenía un efecto sobre el segundo o viceversa y se obtuvieron los resultados que se observan en la Figura 9.

En estos gráficos se aprecia que cuando el primer juego es Trenes, éste es percibido como más fácil $(58.33 \% \mathrm{v} / \mathrm{s} 41.66 \%)(p=0.774)$. Al contrario, cuando el primer juego es Pesca, éste es considerado más fácil que Trenes en una proporción de 1:3 ( $p$ $=0.146$ ).

Independientemente del orden de aplicación de los juegos, el triple de niños considera que el juego Pesca, es más entretenido que Trenes $(p=0.146)$.

Cuando el primer juego aplicado es Trenes, un $83.33 \%$ de los niños señala que se concentra más en Pesca. Esta diferencia es estadísticamente significativa $(p=0.039)$. Cuando el primer juego es Pesca, se mantiene esta tendencia, sin embargo la diferencia es menor entre los juegos: un $58.33 \%$ señala que se concentra más en Pesca y un $41.66 \%$ señala que se concentra más en Trenes $(p=0.774)$.

Al observar el comportamiento de estas variables por condición, se aprecian ciertas diferencias en las percepciones que tuvieron los niños de los distintos grupos. Para más detalles, ver Figura 10. 


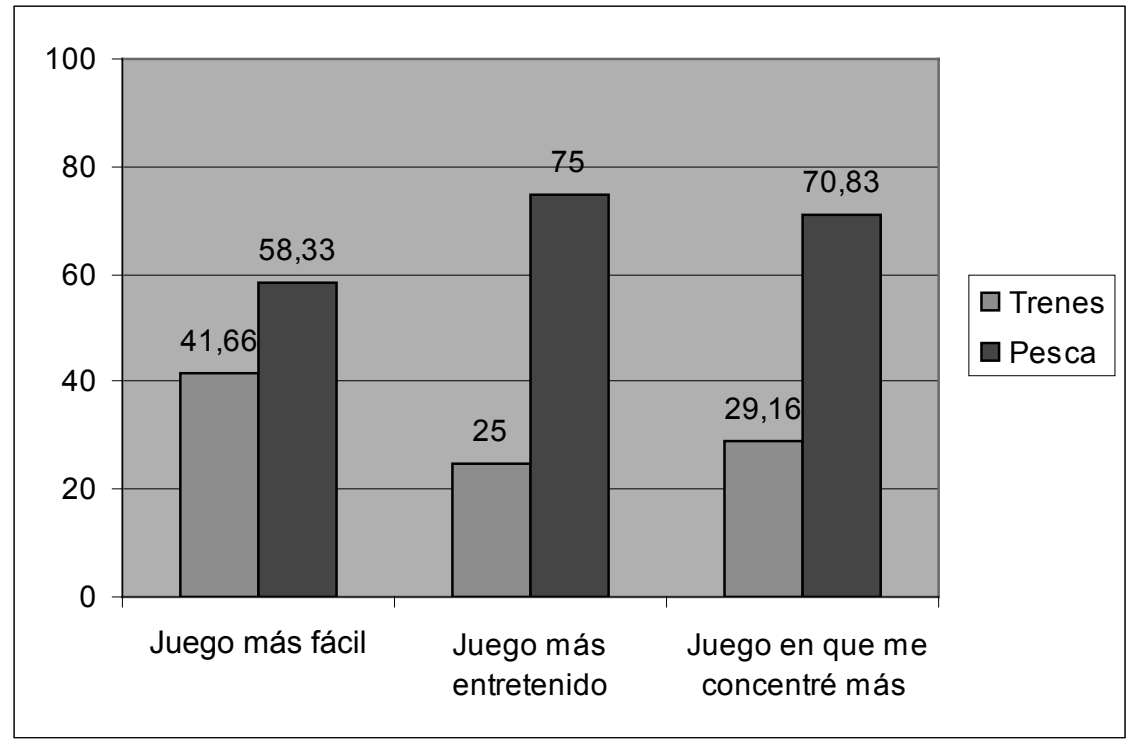

Figura 8. Percepción de los niños respecto a los juegos, en porcentajes.

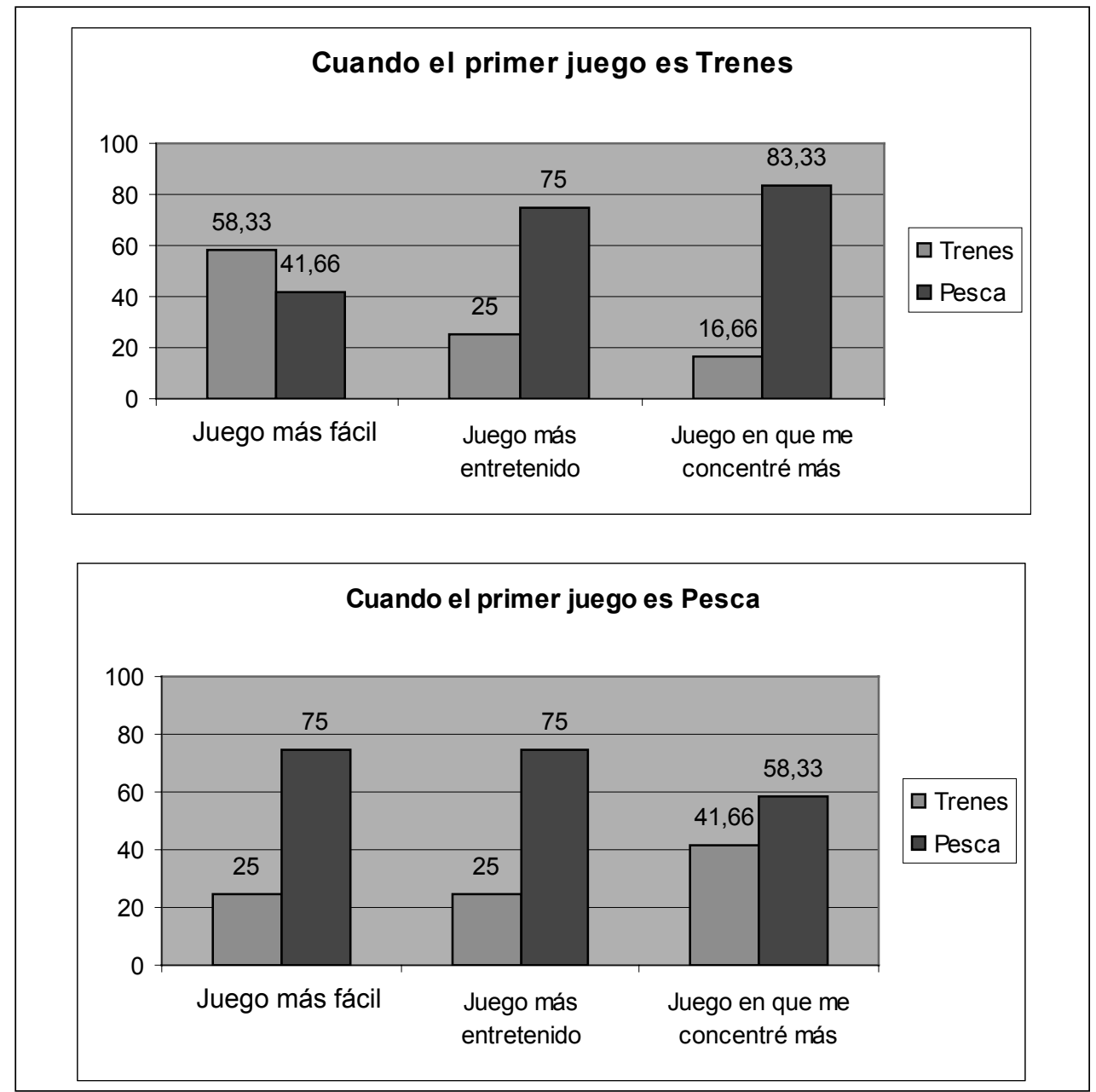

Figura 9. Percepción de los juegos, según el orden de presentación. 


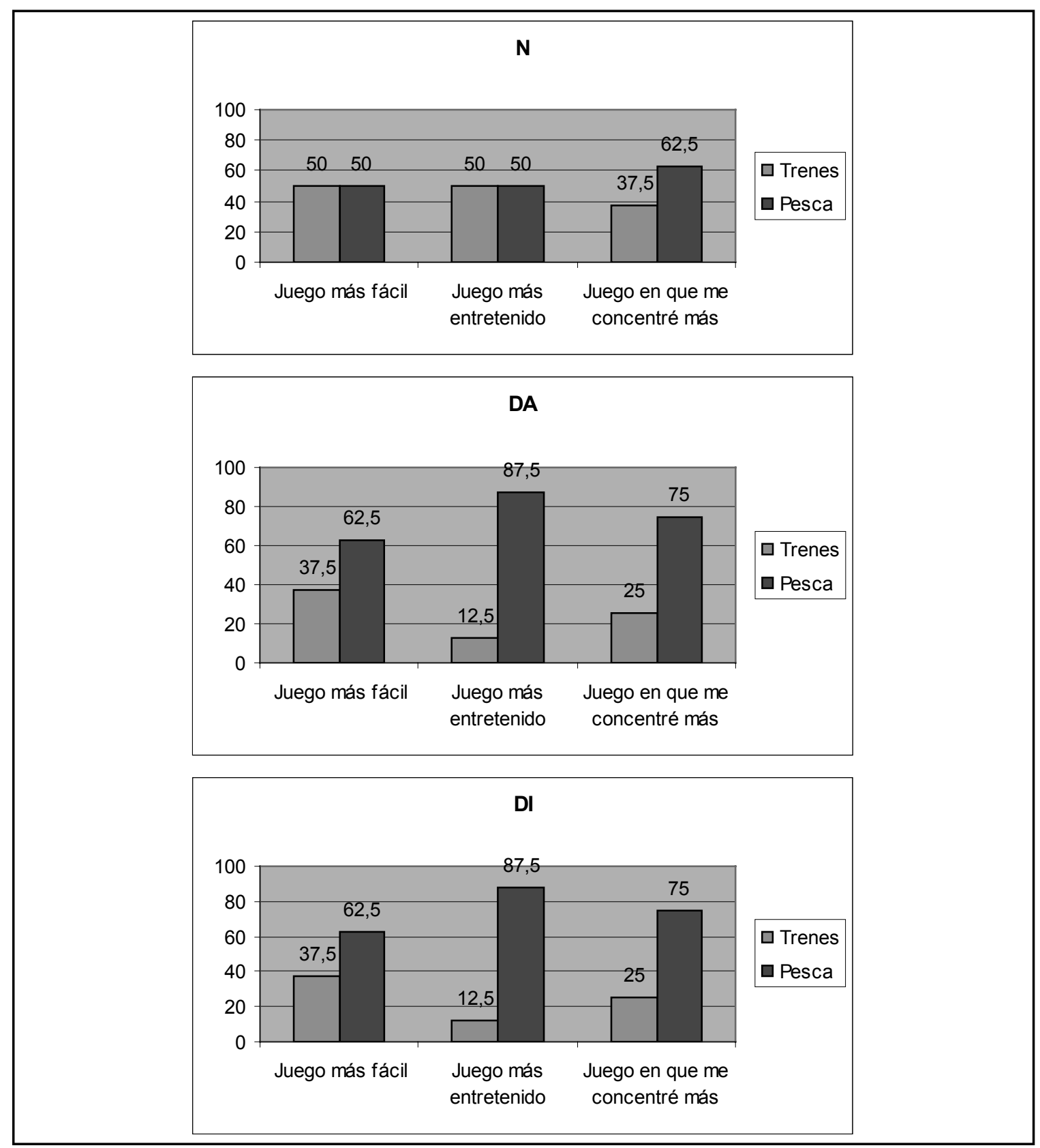

Figura 10. Percepción de los juegos por condición, en porcentajes.

La mitad de los niños $\mathrm{N}$ manifiestan que Trenes es más fácil, mientras la otra mitad considera lo contrario. Lo mismo ocurre la percepción de este grupo respecto a qué juego es más entretenido $(50 \%$ para ambos juegos). Un $62.5 \%$ de los niños $\mathrm{N}$ considera que se concentró más en el juego Pesca que en Trenes. Estas diferencias no son significativas $(p=1.000 ; p=1.000 ; p=0.727)$.

La percepción que tienen los niños DA es similar a la de los niños DI para las tres variables que se están analizando: consideran que el juego Pesca es más fácil que Trenes $(62.5 \% \mathrm{v} / \mathrm{s} 37.5 \%)(p=0.727)$; el juego Pesca le parece más entretenido al $87.5 \%$ de los niños en ambos grupos $(p=0.070)$; y coinciden en que un $75 \%$ de los niños se concentró más en Pesca que en Trenes ( $p=0.289)$.

Por último, se realizó un análisis de correlación entre los aspectos lúdicos y los resultados de evaluación de aprendizaje implícito para ambos juegos, en el cual se observa que existe una asociación esta- 
dísticamente significativa ( $p=0.016)$ entre el juego que los niños perciben más entretenido y aquel que consideran más fácil.

No se encontraron correlaciones entre los aspectos lúdicos de los juegos y el aprendizaje implícito de los niños para ninguno de los juegos.

\section{Conclusiones y Discusión}

\section{¿Es Posible Demostrar Aprendizaje Implícito en Niños?}

Uno de los objetivos que se buscó evaluar con esta investigación fue si el juego creado favorece o no el aprendizaje implícito en niños de entre 6 y 8 años de edad con distintas características (normales, con déficit atencional y con déficit intelectual). Los resultados muestran que el juego creado (Pesca) no promueve especialmente la ocurrencia de aprendizaje implícito, dado que los resultados obtenidos no son significativamente distintos al azar. Este juego tampoco es significativamente distinto al estímulo usado como control. Los resultados generales obtenidos con estos juegos no permiten demostrar fehacientemente la ocurrencia de aprendizaje implícito en niños, lo que concuerda con los resultados obtenidos por Rosas, Condemarín, Nussbaum y Sun (1999) y Rosas y Grau (2002). Por su parte, estos resultados son contradictorios con los reportados por Domuta y Pentek (2003), quienes incluso con una muestra pequeña de niños preescolares refieren la eficacia del paradigma de gramáticas artificiales para demostrar aprendizaje implícito en niños. Existen, no obstante, algunas diferencias en el procedimiento utilizado por estos autores respecto a los usados en esta investigación y en los estudios recién mencionados: a los niños del grupo experimental se les administra la fase de aprendizaje y la fase de evaluación; en cambio, a los niños del grupo control sólo se les administra la fase de evaluación. Las diferencias reportadas son significativas entre ambos grupos y son consistentes con los estudios de Perruchet y Vinter (1998) y de López (2005) que también muestran la eficacia del aprendizaje implícito a través de gramáticas artificiales. De esta manera, las evidencias siguen siendo contradictorias respecto a la demostración de aprendizaje implícito en niños. ¿A qué se pueden deber estas diferencias?

Un aspecto a considerar es que los resultados obtenidos en esta investigación con el juego Tre- nes presentados en el formato computacional, son superiores a los reportados por Grau (2002) en la prueba Trenes original (con un formato de animales de madera 3D). En dicha investigación, tal como sucedió en ésta, no se aprecian correlaciones estadísticamente significativas entre los rendimientos de los juegos aplicados (Maga y Trenes original; Pesca $y$ Trenes, respectivamente). Cabe señalar que para ambos pares de juegos los ejemplares tanto para la fase de aprendizaje como para la fase de evaluación se presentaron en el mismo orden. Estas evidencias llevan a plantearse interrogantes que tienen relación con el formato de presentación de los estímulos.

Si comparamos los dos juegos aplicados es posible reconocer fácilmente que obedecen a la misma gramática y la cantidad de ordenaciones es similar tanto para la fase de aprendizaje como para la fase de evaluación. Sin embargo hay elementos en Pesca que pueden haber afectado el proceso de memorización involucrado en el aprendizaje. Con el fin de hacer más lúdico el juego se incluyeron elementos que en vez de favorecer el aprendizaje, pueden haber interferido la memorización por exceso de elementos distractores. Así por ejemplo, los animales se movían en el fondo del mar, lo que puede haber dificultado la tarea a los niños, sobretodo a los que tenían menor manejo del mouse (en Trenes los animales estaban estáticos). Otro aspecto a considerar se relaciona con los animales elegidos, ya que éstos eran poco familiares para algunos niños de la Región Metropolitana (eran sólo animales marinos), lo que puede haber afectado su memorización. De esta manera, los niños pueden haber ocupado mayor cantidad de recursos atencionales en mover el mouse, o en hacer calzar un nombre a una imagen desconocida, que en aprender la regla subyacente a la gramática en la fase de aprendizaje. No obstante, todos los niños debían realizar todos los ejemplares las veces que fuera necesario para realizarlo correctamente y así poder pasar al siguiente ejemplar. De este modo, estos elementos pueden haber interferido la memorización afectando la motivación por la tarea y por lo tanto, de manera indirecta, los resultados de la evaluación. Pese a ello, los resultados de los niños en la fase de aprendizaje por juego y por condición, no se relacionan con los resultados de la fase de evaluación de aprendizaje implícito. Esto último apoya las ideas de Reber (1993), Seger (1994) y Froufe (1997) quienes indican que el aprendizaje implícito ocurre independientemente de los intentos deliberados por aprender y de la atención dedicada a la tarea. 
A pesar de estos resultados quizás no tan alentadores respecto al formato lúdico, cabe destacar del presente estudio que sí es posible reconocer diferencias por condición frente al aprendizaje implícito: los niños que logran comparativamente mayor aprendizaje son aquellos que presentan déficit atencional, lo cual no deja de ser un hallazgo interesante, especialmente para estos niños que se caracterizan por manifestar dificultades al momento de aprender en contextos educativos formales. El aprendizaje a través de juegos computacionales como éstos podría constituirse en una herramienta de apoyo para la adquisición de reglas subyacentes a gramáticas complejas en niños denominados con déficit atencional.

Otro aspecto a considerar en este estudio consiste en la influencia del orden de presentación de los juegos. No se esperaba que esta variable influyera en los resultados, sin embargo, es posible observar que cuando el orden de presentación es Trenes-Pes$c a$, los niños obtienen un mejor desempeño en el segundo juego. Esta situación se invierte cuando el orden es Pesca- Trenes. Una manera de comprender esta situación puede relacionarse con un efecto de dificultad creciente, en el cual con el primer juego se adquiere una mecánica, la cual se consolida con un segundo juego más complejo y desafiante (que por lo demás consta de más elementos lúdicos que ayudan a mantener la atención). La combinación contraria podría generar un monto excesivo de dificultad inicial que podría llevar al error y progresivamente a la frustración; con el consecuente empobrecimiento de la ejecución en el segundo juego. En este sentido, se puede tratar de un aprendizaje que requiere de más de una sesión de juego y de una combinación particular de formatos lúdicos para alcanzar su máxima potencia.

Por otra parte, en esta investigación se evidenciaron diferencias de ejecución entre los grupos de niños de las distintas condiciones en la segunda aplicación, independientemente del juego utilizado. Los niños normales y los niños con déficit atencional obtuvieron resultados sustantivamente mejores que los niños con déficit intelectual. En este sentido, los resultados son contradictorios con los descritos por Malone y Maybery (1998) quienes sostienen que no hay diferencias en el aprendizaje implícito entre sujetos normales y sujetos con disfunciones neuropsicológicas, apoyando la tesis de la existencia de un sistema más robusto (Reber, 1993; Seger, 1997) que no se vería afectado por el nivel intelectual. Los hallazgos del presente estudio, en cambio, sugieren que niños con problemas de atención aprenden a través de vías implícitas al igual que niños sin problemas de atención y los niños con déficit intelectual presentan un aprendizaje implícito disminuido respecto a los niños de las otras dos condiciones. Ante estas diferencias cabe preguntarse, para cada uno de los casos cuánto de lo que han aprendido o no hasta ahora ha sido influido por esta vía. También surgen otras interrogantes: ¿Qué tan modular es el aprendizaje implícito? ¿De qué manera se relacionan el aprendizaje implícito y el explícito?

\section{¿El Juego es una Herramienta Útil Para Probar Aprendizaje Implícito en Niños?}

Uno de los supuestos teóricos que fundamentó el desarrollo de esta investigación consiste en confiar que el juego es el mejor intermediario para acceder a los niños y que es tan potente, que sirve incluso para superar tareas repetitivas como ocurre en el paradigma de gramáticas artificiales. Por lo tanto era fundamental desarrollar un juego que pudiera ser definido realmente como tal por los niños.

Tal como muestran los resultados, la percepción de los niños en general es que Pesca es más entretenido y en este juego se concentran más que en Trenes. Sin embargo, por condición se aprecian ciertas diferencias: los niños normales presentan una percepción más homogénea entre los juegos. Esto es interesante, ya que sugiere que los niños de este grupo perciben de alguna manera la similitud que hay entre los juegos. Es como si pudieran reconocer de manera más clara que los demás niños, la regla o mecanismo que opera tras las metáforas lúdicas, y anclaran ahí su comparación. Los niños con déficit atencional y con déficit intelectual en cambio, son categóricos en su percepción y señalan que el juego Pesca es más fácil, más entretenido y que en él se concentran más. Estas apreciaciones se relacionan más con las metáforas involucradas para "enmascarar" la gramática y por lo tanto, se relacionan más con el juego que con el paradigma. Este corresponde a un campo especulativo ya que no existen antecedentes en la literatura que den cuenta de esta relación. En general se acepta que la atención focalizada de los sujetos hacia los estímulos es una condición necesaria en el paradigma de gramáticas artificiales (Domuta \& Pentek, 2003) y en ese sentido el juego cumpliría un rol fundamental, ya que favorecería el logro de aprendizaje mediante mayor entretención y concentración. Sin embargo, esto no coincide con los hallazgos de este estudio y 
resulta contradictorio con lo señalado anteriormente respecto a la independencia del "sistema implícito" reportada por Reber (1993), Malone y Maybery (1998) y Grau (2002). Tomando estos aspectos en consideración y pensando en que el paradigma en sí corresponde a una situación experimental, cabe preguntarse seriamente si corresponde evaluar a los niños mediante un formato que intenta cumplir con parámetros lúdicos pero que debe seguir reglas gramaticales que no obedecen necesariamente a lo que Vigotsky (1998) denomina "reglas del juego". ¿Es posible demostrar aprendizaje implícito de una gramática artificial en niños sin que la tarea sea tediosa para ellos? Diversos estudios han demostrado que el aprendizaje implícito operaría plenamente desde la infancia temprana, participando por ejemplo en la adquisición del lenguaje (Gomez \& Gerken, 1999; 2000). Quizás el aprendizaje implícito podría ser demostrado a partir de una gramática natural, tal como ocurre con las gramáticas aplicadas al aprendizaje de idiomas.

Otro tema a revisar consiste en las habilidades requeridas por el juego Pesca, ya que puede ser que sea un formato más apropiado para niños de mayor edad o para niños que manejen habitualmente computadores. Respecto a esto último, se observó en la aplicación que los niños que habían usado previamente computadores, se sentían más confiados y disfrutaban más de los juegos. Los niños que nunca habían usado un computador, en general, tenían problemas en la manipulación de la herramienta y se aburrían antes. Al respecto también sería conveniente evaluar cuál será la cantidad de sesiones óptimas de juego para probar aprendizaje implícito en niños. En investigaciones anteriores (Sun, 2000), se han realizado hasta tres sesiones de entrenamiento de manera tal que los niños puedan familiarizarse con el juego antes de realizar la fase de evaluación. Dado que no conocemos la curva de aprendizaje para los niños de las distintas condiciones, puede ser que los juegos sean una alternativa viable para probar aprendizaje implícito si se encuentra el procedimiento más adecuado para ello. Evidentemente aún queda mucho por investigar respecto al paradigma de gramáticas artificiales, su demostración en niños y sobretodo respecto a la influencia del formato de presentación en los resultados que se reportan en esta población.

\section{Referencias}

Augart, C. (1994). Implicit learning. Psychological Bulletin, $115(2), 163-196$

Baltra, A. (1990). Languaje learning through computer adventure games. Simulation and Gaming, 21, 445-452.

Domuta, A. \& Pentek, I. (2003). Implicit learning in ADHD preschool children. Recuperado el 2 de Julio de 2006, desde el sitio Web de Babes-Bolyai University: http://dppd.ubbcluj. ro/mag/munkatarsak/pentek/implicit.pdf

Froufe, M. (1997). El inconsciente cognitivo: La cara oculta de la mente. Biblioteca Nueva: Madrid.

Gómez, R. L. \& Gerken, L. (1999). Artificial grammar learning by 1-year-old leads to specific and abstract knowledge. Cognition, 70, 109-135.

Gómez, R. L. \& Gerken, L. (2000). Infant artificial lenguage learning and language acquisition. Trends in Cognitive Sciences, 4(5), 178-186.

Grau, V. (2002). Aprendizaje implícito en niños con retardo mental. Relación entre las capacidades de memoria de trabajo $y$ las de aprender de forma inconsciente. Tesis no publicada para optar al grado de Magíster en Psicología Educacional, Pontificia Universidad Católica de Chile, Santiago.

Klawe, M. (1998). When does the use of computers games and other interactive multimedia software help students learn mathematics? Recuperado el 5 de Junio de 2005, desde http:// taz.cs.ubc.ca/egems/papers/UCSMP.doc

Lewicki, P., Czyzewska, M. \& Hoffman, H. (1986). Unconscious acquisition of complex procedural knowledge. Journal of Experimental Psychology: Learning, Memory and Cognition, 13, 523-530.

López, M. F. (2005). Relaciones entre el aprendizaje implícito, explícito e inteligencia en niños de edad escolar. Tesis no publicada para optar al grado de Doctor en Psicología, Universidad Nacional de San Luis, Argentina.

Malone, T. \& Lepper, M. (1987). Making learning fun: A taxonomy for intrinsic motivations for learning. En R. E. Snow \& M. J. Farr (Eds.), Aptitudes, learning and instruction, III: Conative and affective process analysis (pp. 223-253). Hillsdale, NJ: Lawrence Earlbaum Association.

Malone, A. B. \& Maybery, A. (1998). Implicit learning. Implicit and explicit mental processes. New York: Lawrence Erlbaum Associates.

Perruchet, P. \& Vinter A. (1998). Learning and development. En M. Stadler \& P. Frensch (Eds.), Handbook of implicit learning (pp. 495-531). Londres: Sage.

Ramírez, V. \& Rosas, R. (2007). Estandarización del WISC III en Chile: Descripción del test, estructura factorial y consistencia interna de las escalas. Psykhe, 16(1), 91-109.

Reber, A. S. (1967). Implicit learning of artificial grammars. Journal of Verbal Learning and Verbal Behavior, 6, 855-863.

Reber, A. S. (1993). Implicit learning and tacit knowledge. An essay on the cognitive unconscious. New York: Oxford University Press.

Rosas, R., Condemarín, M., Escobar, E., Sun, Y. \& De Padua, E. (1999). Aprendizaje implícito de una gramática artificial en adultos por medio del juego. Manuscrito no publicado, Pontificia Universidad Católica de Chile, Santiago.

Rosas, R., Condemarín, M., Nussbaum, M. \& Sun, Y. (1999). Aprendizaje implícito de una gramática artificial en niños de edad escolar a través de un juego electrónico. Manuscrito no publicado, Pontificia Universidad Católica de Chile, Santiago.

Rosas, R. \& Grau, V. (2002). Aprendizaje implícito y memoria de trabajo: Evidencia para postular su separación funcional. Estudios de Psicología, 23(2), 251-272. 
Rosas, R., Nussbaum, M., Grau, V., López, X., Salinas, M., Flores et al. (2000). Más allá del Mortal Kombat: Diseño y evaluación de videojuegos educativos para lenguaje y matemáticas del nivel básico 1. Psykhe, 9(2), 125-141.

Seger, C. (1994). Implicit learning. Psychological Boulletin, $115,163-196$

Seger, C. (1997). Two forms of sequential implicit learning. Consciousness and Cognition, 6, 108-131.

Stewart, C. \& Kowaltzke, A. (1997). Media: New ways and meanings. Brisbane: Jacaranda Wiley.

Fecha de recepción: enero de 2007.

Fecha de aceptación: julio de 2007.
Sun, Y. (1998). Aprendizaje implícito: Aspectos críticos de su definición y algunas de sus implicancias. Psykhe, 7(2), 41-51.

Sun, Y. (2000). Aprendizaje implícito de una gramática artificial en niños en edad escolar por medio de un juego electrónico. Tesis no publicada para optar al grado de Magíster en Psicología Educacional, Pontificia Universidad Católica de Chile, Santiago.

Vigotsky, L. (1998). El desarrollo de procesos psicológicos superiores. México: Grijalbo. 